\title{
Nsp7 and Spike Glycoprotein of SARS-CoV-2 are envisaged as Potential Targets of Vitamin D and Ivermectin
}

Jhimli Dasgupta ${ }^{1, \#}$, Udayaditya Sen ${ }^{2, \#}$, Abhisek Bakshi ${ }^{3}$, Abhijit Dasgupta $^{4}$, Krishnendu Manna $^{5}$, Chinmay Saha ${ }^{6}$, Rajat K. De ${ }^{7}$, Satinath Mukhopadhyay ${ }^{8}$, Nitai P. Bhattacharyya ${ }^{8 *}$.

${ }^{1}$ Department of Biotechnology, St. Xavier's College, 30 Park Street, Kolkata 700016, India.

${ }^{2}$ Crystallography and Molecular Biology Division, Saha Institute of Nuclear Physics, HBNI, 1/AF Bidhan Nagar, Kolkata, 700064, India.

${ }^{3}$ Department of Information Technology, Bengal Institute of Technology, Basanti Highway, Kolkata 700150, West Bengal, India.

${ }^{4}$ Department of Data Science, School of Interdisciplinary Studies, University of Kalyani, Kalyani, Nadia 741235, West Bengal, India.

${ }^{5}$ Department of Food \& Nutrition, University of Kalyani, Kalyani, Nadia 741235, West Bengal, India.

${ }^{6}$ Department of Genome Science, School of Interdisciplinary Studies, University of Kalyani, Kalyani, Nadia 741235, West Bengal, India.

${ }^{7}$ Machine Intelligence Unit, Indian Statistical Institute, 203 B.T. Road, Kolkata 700108, India.

${ }^{8}$ Department of Endocrinology and Metabolism, Institute of Post Graduate Medical Education \& Research and Seth Sukhlal Karnani Memorial Hospital, Kolkata 700020, West Bengal, India.

*Correspondence should be addressed to Nitai P. Bhattacharyya, Ph.D, Department of Endocrinology and Metabolism, Institute of Post Graduate Medical Education \& Research and Seth Sukhlal Karnani Memorial Hospital, Kolkata 700020, West Bengal, India. email Id: nitai.bhattacharyya@gmail.com

\# Equal contribution

Keywords: SARS-CoV-2, Vitamin D, Ivermectin, RNA-dependent-RNA polymerase, Spike glycoprotein, Knowledge based docking 


\section{Abstract}

COVID-19 has emerged as deadly pandemic worldwide with no vaccine or suitable antiviral drugs to prevent or cure the disease. Because of the time-consuming process to develop new vaccines or antiviral agents, there has been a growing interest in repurposing some existing drugs to combat SARS-CoV-2. Vitamin D is known to be protective against acute respiratory distress syndrome (ARDS), pneumonia and cytokine storm. Recently it has been used as a repurposed drug for the treatment of H5N1 virus-induced lung injury. Circumstantial evidences indicate that people with low level of vitamin $D$ are more susceptible to SARS-CoV-2. Although, vitamin D was suggested to interfere with viral replication, its interaction with any SARS-CoV-2 protein is unexplored yet. Beside this, ivermectin, a well-known anti-parasitic agent, exhibits potent anti-viral activities in vitro against viruses such as HIV1 and dengue. Very recently, ivermectin has been found to reduce viral load of SARS-CoV-2 in vitro. We have analyzed available structures of SARS-CoV-2 proteins to identify probable binding partner(s) of vitamin $\mathrm{D}$ and ivermectin through knowledge-based docking studies and figured out possible implication of their binding in SARS-CoV-2 infection. Our observations suggest that the non-structural protein nsp7 possesses a potential site to house 25-hydroxyvitamin D3 (VDY) or the active form of Vitamin D, calcitrol. Binding of vitamin D with nsp7 likely to hamper the formation of nsp7-nsp8 complex which is required to bind with RNA dependent RNA polymerase (RdRP), nsp12 for optimal function. On the other hand, potential binding site of ivermectin has been identified in the S2 subunit of trimeric spike(S) glycoprotein of SARS-CoV-2. We propose that deeply inserted mode of ivermectin binding at three inter-subunit junctions may restrict large scale conformational changes of S2 helices which is necessary for efficient fusion of viral and host membrane. Our study, therefore, opens up avenues for further investigations to consider vitamin $D$ and ivermectin as potential drugs against SARS-COV-2. 


\section{Introduction}

The COVID-19 pandemic has led to widespread loss of human lives and has impacted severely on global economy. With no herd immunity, either natural or vaccine induced, prevention of human to human transmission by monitoring or containment through lockdown seems to be the only effective tool to halt the progress of this pandemic. Since vaccines or new antiviral agents will take time to develop, there has been a growing interest in repurposing some existing drugs to prevent the entry and/or replication of the virus into the host or to blunt the cytokine storm that ultimately leads to mortality. Several drugs are undergoing evaluation in the quest for finding an effective and safe agent to treat the COVID-19 or severe acute respiratory syndrome coronavirus-2 (SARS-CoV-2) infection.

So far, RNA-dependent RNA polymerase (RdRp) of SARS-CoV-2 remained as a promising drug target due to its crucial role in RNA synthesis and absence of its homolog in the host (Gao et al., 2020). Different nucleos(t)ide analogues (NAs) are being used as viral RdRp inhibitors (Gao et al., 2020) since metabolized form of the NAs are subsequently incorporated into the nascent viral RNA by the RdRp, accounting for the antiviral effect. Through modelling studies, several anti-polymerase drugs like Ribavirin, Remdesivir, Sofosbuvir, Galidesivir, and Tenofovir were proposed to bind with RdRp of SARSCoV-2, (Elfiky et al., 2020, Gao et al., 2020). While this method is useful for several viruses it is particularly challenging for SARS-CoV-2 due to the exonuclease activity of non-structural proteins nsp14 which is capable of excising incorporated NAs (Shannon et al., 2020).

Apart from RdRP, several other SARS-CoV-2 proteins are therefore being studied as possible drug targets. Among them, 3-chymotrypsin-like cysteine protease 3 (3CLpro) and papain like protease (PLpro), involved in the proteolytic processing of the viral polyprotein, are proven as drug target in the case of SARS-CoV and middle east respiratory syndrome coronavirus (MERS-CoV). Several FDA approved anti-viral drugs as well as traditional Chinese medicine and natural products were identified which could serve as potential anti SARS-CoV-2 lead molecules (Wu et al., 2020, UI Qamar et al. 2020).

Recently, Vitamin $D$ has been shown to be protective against infections associated with acute respiratory distress syndrome (ARDS), pneumonia and cytokine storm (Tsujino et al., 2019, Zhou et al., 2019, Hong et al., 2020). Vitamin D has been proposed as a repurposed drug for treatment of H5N1 virus-induced lung injury (Huang et al., 2020). In fact, there are some circumstantial evidences to show that vitamin D may be useful for controlling COVID-19, like many other viral infections (Grant et al., 2020). Although the protective effect of vitamin D against COVID-19 is more likely related to suppression of cytokine response and reduced severity/risk for ARDS, there is also evidence from a meta-analysis that regular oral vitamin D2/D3 intake is safe and protective against acute respiratory tract infection, especially in case of vitamin D deficiency (Martineau et al., 2017). However, it is not 
yet known whether vitamin D interacts with any of the SARS-CoV-2 proteins. Teymoori-Rad et al., 2019 has suggested that antiviral effects of vitamin D can either interfere with viral replication or can act in an immunomodulatory and anti-inflammatory way. Availability of structures of many proteins coded by SARS-CoV-2 opens up possibility of virtual screening of available drugs, including vitamin $D$, for repurposing.

Ivermectin is a well-known anti-parasitic agent that exhibits potent anti-viral activities against a broad range of viruses in vitro such as Venezuelan Equine Encephalitis Virus, HIV-1 and dengue virus (Gotz, V., et al, 2016; Lundberg, L., et al, 2013; Tay, M.Y., et al, 2013; Wagstaff, K.M., et al.,2012). Very recently, Caly et al. (2020) has shown in vitro that ivermectin acts as an inhibitor of SARS-CoV-2. Addition of $5 \mu \mathrm{M}$ of ivermectin to Vero-hSLAM cells 2 hours post infection with SARS-CoV-2 able to cause $\sim 5000$-fold reduction in viral RNA at $48 \mathrm{~h}$ (Caly et al., 2020). Although ivermectin was expected to be an inhibitor of importin alpha/beta (IMPa-IMPß1) mediated nuclear import in case of HIV-1 (Tay et al., 2013; Wagstaff et al., 2012; Yang et al., 2020), this is not significant to limit the viral load of SARS-CoV-2, since no nuclear import is reported so far for the proliferation of this virus. Reduction of RNA load of SARS-CoV-2 observed in the presence of ivermectin therefore judiciously points towards its possible inhibitory effect on the structural and/or non-structural protein(s). Ivermectin therefore warrants further inspection to identify its possible protein target in SARS-CoV-2. In the current study, we explore, with the help of knowledge-based docking strategies, the potential antiviral properties of vitamin $\mathrm{D}$ and ivermectin, to identify their possible role in preventing and/or treating this pandemic.

\section{Results and Discussion}

\section{Binding of vitamin D with nsp7 and its implication of RdRP function}

We have explored any probable binding site of vitamin D with the replication/transcription machinery of SARS-CoV-2. Structural and biochemical studies demonstrated that RdRP (nsp12) is the key component that catalyzes the synthesis of viral RNA while nsp7 and nsp8 act as essential cofactors. Subissi et al (2014) showed that SARS-CoV nsp12 alone is minimally active and its activity is highly dependent on nsp7 and nsp8. Neither nsp7 nor nsp8 alone is sufficient for optimal function of nsp12 but nsp7-nsp8 complex confers processivity to the RNA synthesizing activity of nsp12 (Subissi et al, 2014). Structure of RdRP of SARS-CoV-2 (Gao et al., 2020), revealed that nsp12 binds with nsp7-nsp8 dimer and another nsp8 monomer to be optimally active, which is in accordance with the RdRP structure of SARS-CoV (Kirchdoerfer et al, 2019). Structure of nsp12-nsp7-nsp8 complex of SARS-CoV demonstrated that the binding of the nsp7-nsp8 heterodimer to the index finger loop of nsp12 stabilizes the polymerase domain of the latter to permit RNA template recognition (Kirchdoerfer et al, 
2019). Nsp12 of SARS-CoV and SARS-CoV-2 share a remarkable $96 \%$ sequence identity (Morse et al. 2020; Gao et al., 2020). Similarly, sequences of nsp7 and nsp8 of SARS-CoV and SARS-CoV-2 are also highly conserved, having $99 \%$ and $97.5 \%$ identities respectively (Fig. 1a,b). Understandably, formation and binding of nsp7-nsp8 complex to nsp12 is essential for the optimal activity of RdRP of SARS-CoV2.

Our inspection of the structure of human serum vitamin D-binding protein in complex with a vitamin D3 metabolite 25-hydroxyvitamin D3 (VDY) revealed that VDY binds in a hydrophobic cleft formed between two helices arranged in an elbow like pattern (PDB code: 1J78, Verboven et al, 2002). Interestingly, inspection of SARS-CoV-2 RdRP complex structure (PDB code: 7BTF) identified similar hydrophobic pocket in the cofactor nsp7 which is reminiscent of the binding of VDY to human serum vitamin D-binding protein (Verboven et al, 2002). In RdRP-cofactor complex (nsp12-nsp7-nsp8) of SARS-CoV-2, this pocket of nsp7 remains occupied by the $\mathrm{N}$-terminal helix of nsp8 through hydrophobic packing (Fig. 2a). We have docked VDY with nsp7 considering surface charge and groove complementarity (Fig. 2b). Polar interaction of VDY with Thr9 of nsp7 along with hydrophobic packing with nsp7 residues Leu13, Leu17, Leu20, Phe49 and Val53 thus point towards productive binding of VDY with nsp7 (Fig. 2b, 2c). Calculation by PDBePISA (Krissinel et al., 2007) suggested a buried surface area of $410 \AA^{2}$ for nsp7-VDY complex which is comparable with the area of $440 \AA^{2}$ for human serum vitamin D-binding protein-VDY complex.

Residues of nsp7, involved in VDY binding, are also conserved in SARS-CoV, Bat Coronavirus (BtCoV) and MERS coronavirus (Fig. 1a). The mode of interaction of VDY with nsp7, proposed in our modeling study, will certainly hamper the formation of nsp7-nsp8 complex. Since binding of this cofactor complex to nsp12 is essential for its finger loop stabilization followed by efficient recognition of RNA, binding of VDY to nsp7 is expected to be detrimental to the functionality of SARS-CoV-2 RdRP (Fig. 2d). Calcitrol, the active form of Vitamin D, having one extra hydroxyl group at C1 position with respect to VDY, could also be docked at the same site of nsp7 without any hindrance. Therefore, calcitrol can also be proposed as a potential drug to hinder the formation of functional RdRP assembly of SARS-CoV-2.

In the clinical context, however, it remains to be seen if vitamin $D$ deficiency predisposes to SARS-CoV-2 infection and if so, whether vitamin D supplementation would prevent a potential viral outbreak. It is also unknown whether to prevent SARS-CoV-2 binding competitively, one needs to maintain serum vitamin $D$ levels within the normal range or one needs to have a pharmacological dose of vitamin $D$ to achieve this goal. 


\section{Ivermectin binding with spike (S) glycoprotein may impair viral infection}

To search for the target protein and potential binding site of ivermectin, we have systematically analyzed available structures of SARS-CoV-2 and SARS-CoV proteins. Coordinates of SARS-CoV-2 spike protein ectodomain (PDB code:6VYB, 6VXX), nsp12-nsp7-nsp8 (PDB code: 7BTF), protease (PDB code: $5 R 8 T$ ), post fusion core S2 subunit (PDB code: 6LXT), ADP Ribose Phosphatase nsp3 along with SARS-CoV complex nsp14-nsp10 were retrieved for this purpose. Ivermectin acts as a positive allosteric modulator of transmembrane pentameric Cys-loop receptors such as glutamategated chloride channels (GluCls), $\gamma$-aminobutyric acid receptors, glycine receptors (GlyRs) (Hibbs et al., 2011; Huang et al., 2017). Structure of human GlyRa3 indicated that ivermectin binds in a deep cleft made of three helices located at the subunit interface (PDB code: 5VDH). Guided by this information, we have critically analyzed the SARS-CoV-2 protein structures to search for probable ivermectin binding site(s). Interestingly, we have identified a deep cleft in spike(S) protein ectodomain (PDB code: 6VYB, Walls et al., 2020) which is similar to that observed in GlyRa3-ivermectin complex (Fig. 3a,b). S protein is a homotrimeric transmembrane glycoprotein protruding from the viral surface (Tortorici and Veesler, 2019). Its S1 subunit forms the crown and binds with host cell receptor while its S2 subunit is responsible for membrane fusion. Ivermectin binding cleft was observed at the interface of two S2 subunits (Fig. 3b) implying that three such ivermectin binding sites exist in the trimer. Ivermectin could be properly docked in each pocket satisfying size and charge compatibility (Fig. 3b). The benzofuran head of ivermectin which is orientated toward the deep cleft of the binding pocket can be stabilized by polar interactions with Glu725 or Lys1028 of the same chain of the trimer (Fig. 3c). Furthermore, quite a few hydrophobic residues and non-polar parts of the polar residues are found to contribute to the packing of ivermectin with S2 subunit (Fig. 3c). The surface area of S glycoprotein becomes buried upon binding of each ivermectin molecule has been calculated by PDBePISA (Krissinel et al., 2007). The area is of about $600 \AA^{2}$ which is comparable to the buried surface area of human GlyR $\alpha 3$ upon ivermectin binding $\left(670 \AA^{2}\right)$. The free energy of stabilization upon ivermectin binding to SARS-CoV-2 spike (S) protein is $-15 \mathrm{kCal} / \mathrm{mole}$ as calculated by PDBePISA.

Additionally, we have analyzed the mutations found in the spike (S) glycoprotein of SARS-CoV2 isolated from different territories with respect to China Wuhan isolate (Fig. 4a). Mapping of those mutations on the closed state of $\mathrm{S}$ protein ectodomain structure revealed that none of those mutations is located near the proposed ivermectin binding site (Fig. 4b). This implies that binding of ivermectin will not be affected by these mutations. 
As observed by Walls et al., 2020 (and in their previous studies) that removal of the trimeric S1 crown coupled with large scale conformational changes in S2 allows fusion of viral and host membrane for all corona viruses. In highly pathogenic coronaviruses, S glycoprotein trimers are found in partially opened states, while they remain largely closed in human coronaviruses associated with common colds (Walls et al., 2020). They further hypothesized that in the case of SARS-CoV-2, SARS$\mathrm{COV}$ and MERS-CoV, S glycoprotein trimers spontaneously samples between closed and open conformations for optimal functioning. Our proposed ivermectin binding site is located at the interface of two $\mathrm{S} 2$ subunits which is also not too far from the S1 crown. We presume that deeply inserted mode of ivermectin binding at three inter-subunit junction and its sheer size, may disallow the S2 helices to undergo large scale conformational changes necessary for efficient fusion of viral and host membrane. Furthermore, binding of ivermectin may change the micro-environment that restricts the conformational switching between closed and open states of S1, thereby inhibiting SARS-CoV-2 infection. Our observations, therefore, warrant further investigations, on urgent basis, at in vitro and in vivo levels to consider ivermectin as a possible drug of SARS-CoV-2.

\section{Methods}

We have performed graphical inspection of the surface of nsp7, nsp8 and nsp12 for binding pockets of 25-hydroxyvitamin D3 (VDY) (whose coordinates were retrieved from PDB code: 1J78). A suitable binding pocket appeared on nsp7 which resembles of the binding pocket of VDY on human serum vitamin D-binding protein. A knowledge-based docking of VDY was performed based on surface charge and size compatibility in the pocket of nsp7 using COOT (Emsley and Cowtan, 2004) and PyMOL (DeLano Scientific LLC) followed by general energy minimization. Contacts with the surrounding residues were judged graphically in PyMOL and using CONTACT program in CCP4 suite. Similar search was executed for ivermectin binding pocket on the SARS-CoV-2 proteins using the ivermectin coordinates retrieved from the crystal structure of Human Glycine Receptor alpha-3 bound to ivermectin (PDB code: 5VDH). After finding a suitable binding pocket on spike (S) glycoprotein docking of the drug has been carried out using the method described above. Buried surface areas for both the docked complexes are calculated using PDBePISA. However, during docking, we haven't changed the coordinates of protein atoms to consider induced-fit of protein upon ligand binding that causes tighter packing of the ligand.

Graphical presentations are prepared using PyMOL. Sequences of nsp7 and nsp8 of different sources were aligned by MULTALIN (Corpet, 1988) that uses hierarchical clustering using the matrix BLOSUM62, with gap weight -12 and gap length weight -2 . 


\section{References}

Gao Y, Yan L, Huang Y, Liu F, Zhao Y, Cao L, Wang T, Sun Q, Ming Z, Zhang L, Ge J, Zheng L, Zhang $Y$, Wang $\mathrm{H}$, Zhu Y, Zhu C, Hu T, Hua T, Zhang B, Yang X, Li J, Yang H, Liu Z, Xu W, Guddat LW, Wang Q, Lou Z, Rao Z. Structure of the RNA-dependent RNA polymerase from COVID-19 virus. Science. 2020 Apr 10. pii: eabb7498. doi: 10.1126/science.abb7498.

Elfiky AA, Ribavirin, Remdesivir, Sofosbuvir, Galidesivir, and Tenofovir against SARS-CoV-2 RNA dependent RNA polymerase (RdRp): A molecular docking study, Life Sci. 2020 Mar 25;253:117592.

Shannon A, Le NT, Selisko B, Eydoux C, Alvarez K, Guillemot JC, Decroly E, Peersen O, Ferron F, Canard B. Remdesivir and SARS-CoV-2: Structural requirements at both nsp12 RdRp and nsp14 Exonuclease active-sites. Antiviral Res. 2020 Apr 10;178:104793. doi: 10.1016/j.antiviral.2020.104793.

Wu C, Liu Y, Yang Y, Zhang P, Zhong W, Wang Y, Wang $Q$, Xu Y, Li M, Li X, Zheng $M$, Chen L, Li $\mathrm{H}$, Analysis of therapeutic targets for SARS-CoV-2 and discovery of potential drugs by computational methods, Acta Pharm Sin B. 2020 Feb 27. doi: 10.1016/j.apsb.2020.02.008. [Epub ahead of print]

UI Qamar MT, Alqahtani SM, Alamri MA, Chen LL, Structural basis of SARS-CoV-2 3CLpro and anti-COVID-19 drug discovery from medicinal plants. J Pharm Anal. 2020 Mar 26. doi: 10.1016/j.jpha.2020.03.009. [Epub ahead of print]

Tsujino I, Ushikoshi-Nakayama R, Yamazaki T, Matsumoto N, Saito I. Pulmonary activation of vitamin D3 and preventive effect against interstitial pneumonia. J Clin Biochem Nutr 65: 245-251, 2019.

Zhou YF, Luo BA, Qin LL. The association between vitamin D deficiency and communityacquired pneumonia: A meta-analysis of observational studies. Medicine (Baltimore) 98: e17252, 2019

Hong $\mathrm{M}$, Xiong $\mathrm{T}$, Huang J, Wu Y, Lin L, Zhang Z, Huang L, Gao D, Wang H, Kang C, Gao Q, Yang $X$, Yang N, Hao L. Association of vitamin D supplementation with respiratory tract infection in infants. Matern Child Nutr 5: e12987, 2020.

Huang F, Zhang C, Liu Q, Zhao Y, Zhang Y, Qin Y, Li X, Li C, Zhou C, Jin N, Jiang C. Identification of amitriptyline $\mathrm{HCl}$, flavin adenine dinucleotide, azacitidine and calcitriol as repurposing drugs for influenza A H5N1 virus-induced lung injury. PLoS Pathog 16: e1008341, 2020.

Grant WB, Lahore H, McDonnell SL, Baggerly CA, French CB, Aliano JL, Bhattoa HP, Evidence that Vitamin D Supplementation Could Reduce Risk of Influenza and COVID-19 Infections and Deaths, Nutrients. 2020 Apr 2;12(4). pii: E988.

Martineau AR, Jolliffe DA, Hooper RL, et al. Vitamin D supplementation to prevent acute respiratory tract infections: systematic review and meta-analysis of individual participant data. BMJ. 2017;356:i6583.

Teymoori-Rad M, Shokri F, Salimi V, Marashi SM. The interplay between vitamin D and viral infections. Rev Med Virol. 2019 Mar;29(2):e2032. doi: 10.1002/rmv.2032.

Götz V, Magar L, Dornfeld D, Giese S, PohImann A, Höper D, Kong BW, Jans DA, Beer M, Haller $O$, Schwemmle $M$. Influenza A viruses escape from MxA restriction at the expense of efficient nuclear VRNP import. Sci Rep. 2016 Mar 18;6:23138. doi: 10.1038/srep23138.

Lundberg L, Pinkham C, Baer A, Amaya M, Narayanan A, Wagstaff KM, Jans DA, Kehn-Hall K. Nuclear import and export inhibitors alter capsid protein distribution in mammalian cells and reduce Venezuelan Equine Encephalitis Virus replication. Antiviral Res. 2013 Dec;100(3):662-72. doi: 10.1016/j.antiviral.2013.10.004.

Tay MY, Fraser JE, Chan WK, Moreland NJ, Rathore AP, Wang C, Vasudevan SG, Jans DA. Nuclear localization of dengue virus (DENV) 1-4 non-structural protein 5; protection against all 4 DENV serotypes by the inhibitor Ivermectin. Antiviral Res. 2013 Sep;99(3):301-6. doi: 10.1016/j.antiviral.2013.06.002.

Wagstaff KM, Sivakumaran $H$, Heaton $S M$, Harrich $D$, Jans DA. Ivermectin is a specific inhibitor of importin $\alpha / \beta$-mediated nuclear import able to inhibit replication of HIV-1 and dengue virus. Biochem J. 2012 May 1;443(3):851-6. doi: 10.1042/BJ20120150. 
Caly L, Druce JD, Catton MG, Jans DA, Wagstaff KM. The FDA-approved drug ivermectin inhibits the replication of SARS-CoV-2 in vitro. Antiviral Res. 2020 Apr 3;178:104787. doi: 10.1016/j.antiviral.2020.104787.

Subissi L, Posthuma CC, Collet A, Zevenhoven-Dobbe JC, Gorbalenya AE, Decroly E, Snijder EJ, Canard B, Imbert I. One severe acute respiratory syndrome coronavirus protein complex integrates processive RNA polymerase and exonuclease activities. Proc Natl Acad Sci U S A. 2014 Sep 16;111(37):E3900-9. doi: 10.1073/pnas.1323705111.

Kirchdoerfer RN, Ward AB. Structure of the SARS-CoV nsp12 polymerase bound to nsp7 and nsp8 co-factors. Nat Commun. 2019 May 28;10(1):2342. doi: 10.1038/s41467-019-10280-3.

Verboven C, Rabijns A, De Maeyer M, Van Baelen H, Bouillon R, De Ranter C. A structural basis for the unique binding features of the human vitamin D-binding protein. Nat Struct Biol. 2002 Feb;9(2):131-6.

Krissinel E, Henrick K. Inference of macromolecular assemblies from crystalline state. J Mol Biol. 2007 Sep 21;372(3):774-97.

Hibbs RE, Gouaux E. Principles of activation and permeation in an anion-selective Cys-loop receptor. Nature. 2011 Jun 2;474(7349):54-60. doi: 10.1038/nature10139.

Huang X, Chen H, Shaffer PL. Crystal Structures of Human GlyRa3 Bound to Ivermectin. Structure. 2017 Jun 6;25(6):945-950.e2. doi: 10.1016/j.str.2017.04.007.

Walls AC, Park YJ, Tortorici MA, Wall A, McGuire AT, Veesler D. Structure, Function, and Antigenicity of the SARS-CoV-2 Spike Glycoprotein. Cell. 2020 Apr 16;181(2):281-292.e6. doi: 10.1016/j.cell.2020.02.058.

Tortorici MA, Veesler D. Structural insights into coronavirus entry. Adv Virus Res. 2019;105:93116. doi: 10.1016/bs.aivir.2019.08.002.

Emsley P, Cowtan K. Coot: model-building tools for molecular graphics. Acta Crystallogr D Biol Crystallogr. 2004 Dec;60(Pt 12 Pt 1):2126-32.

Corpet F. Multiple sequence alignment with hierarchical clustering. Nucleic Acids Res. 1988 Nov 25;16(22):10881-90.

Competing Interests statement: None declared. 


\section{Figures and Figure legends}

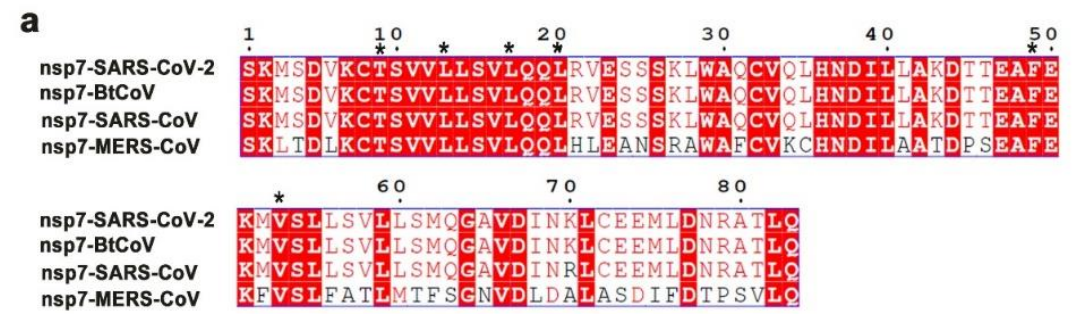

b
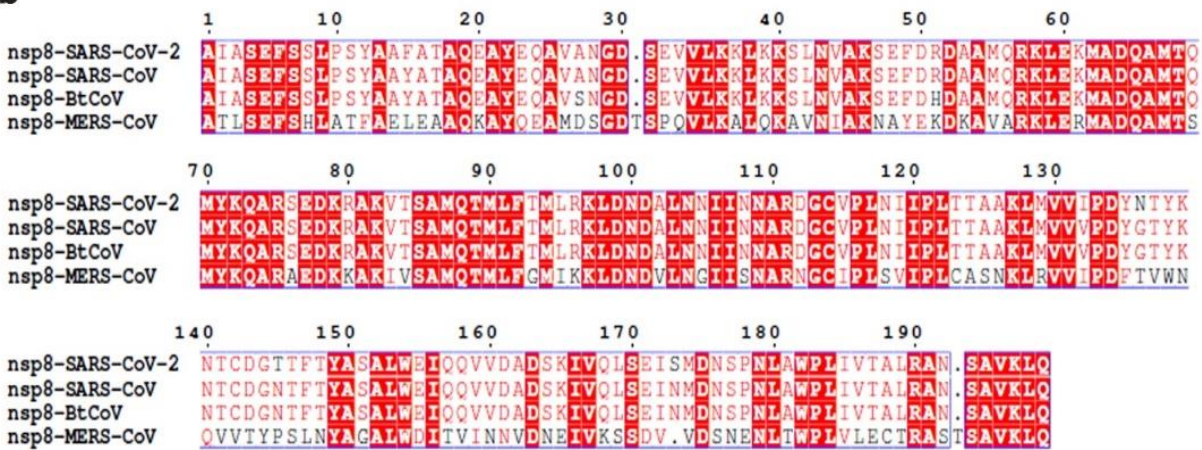

Figure 1: Sequence alignment of (a) nsp7 and (b) nsp8 of SARS-CoV-2 with the sequences of SARSCoV, Bat Coronavirus (BtCoV) and Middle East Coronavirus (MERS); The conserved amino acids of nsp7 which may show probable interaction with vitamin D are marked by '*' in (a). 
a

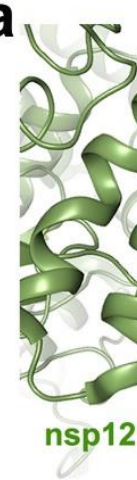

C

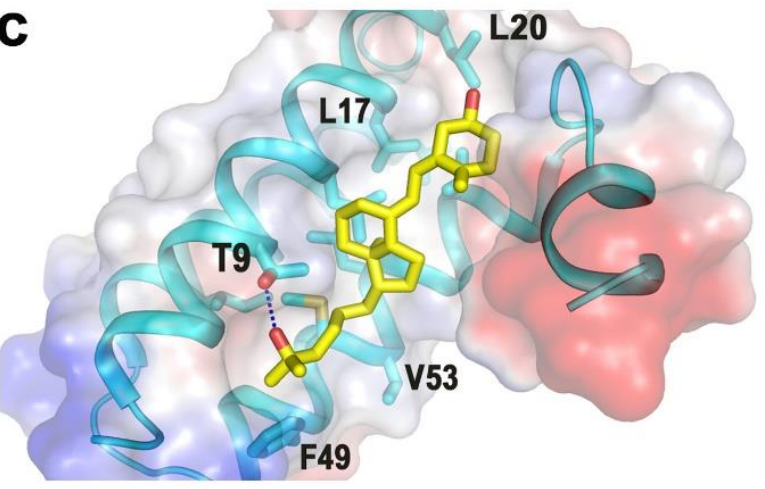

b

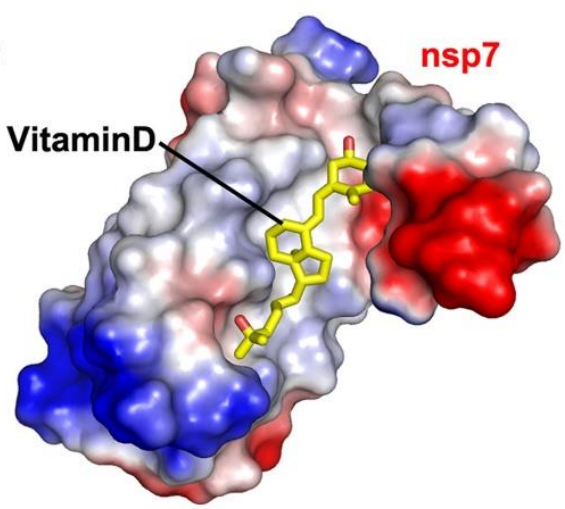

d

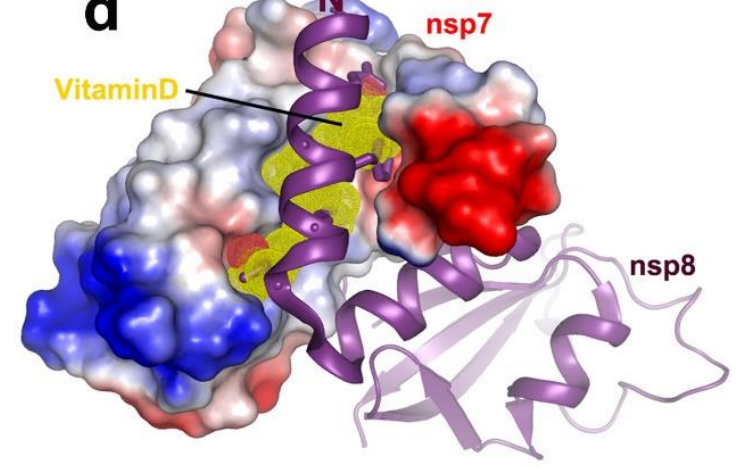

Figure 2: (a) Structure of nsp12-nsp7-nsp8 complex of SARS-CoV-2; (b) electrostatic surface of nsp7 with docked vitamin D (shown as yellow sticks); (c) probable polar and hydrophobic interactions between nsp7 and vitamin D in the docked complex; (d) Vitamin D will impair binding of nsp8 with nsp7. 


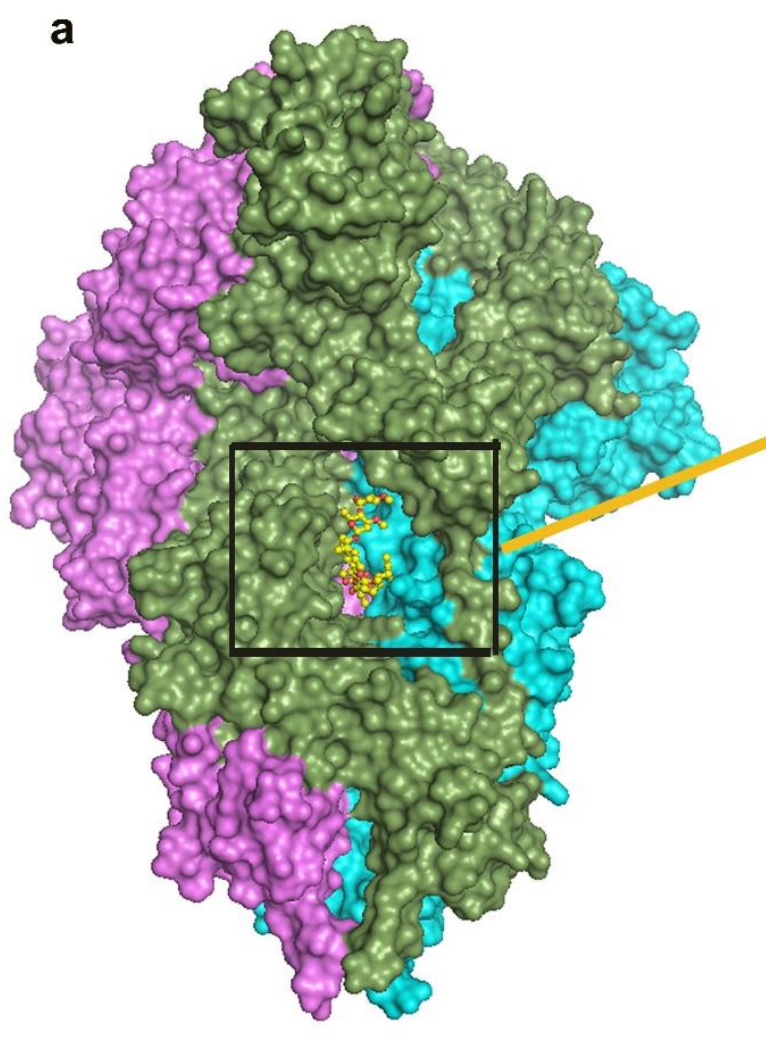

b

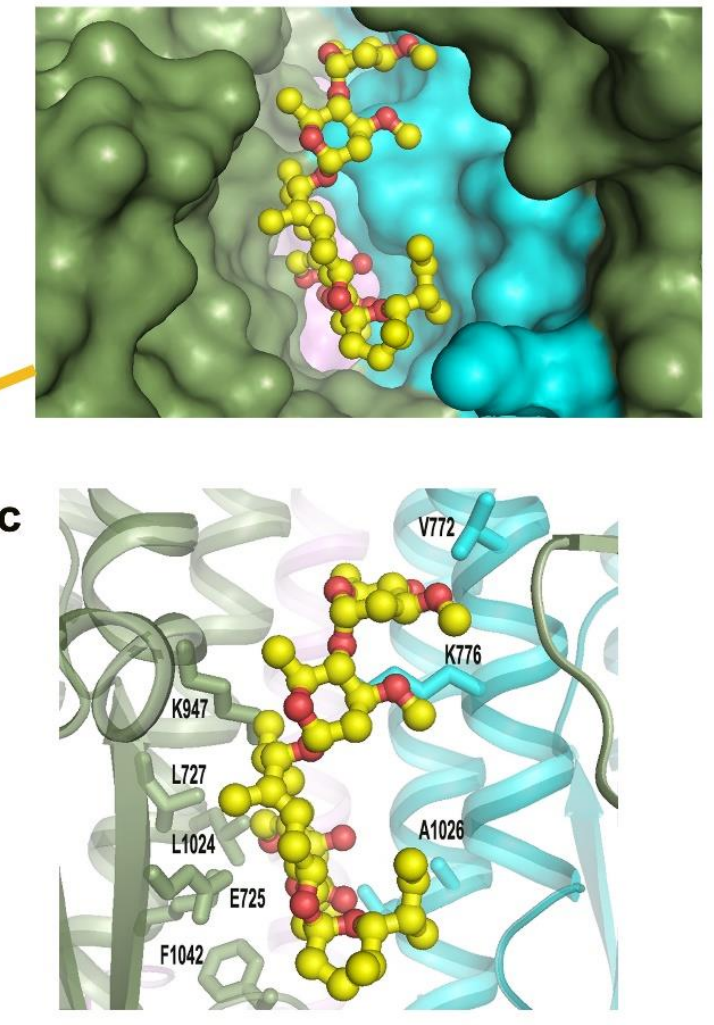

Figure 3: (a) Ivermectin (shown in yellow ball and stick) docked in homotrimeric transmembrane spike (S) protein of SARS-CoV-2; (b) zoomed view of (a) showing fitting of ivermectin in the inter-subunit cleft of S protein; (c) probable interactions between ivermectin and S protein. 
a

\begin{tabular}{|l|c|l|}
\hline SARS-CoV-2 Spike Protein & \multicolumn{2}{|c|}{$\begin{array}{c}\text { Mutations with respect to } \\
\text { ChinaWuhan Isolate }\end{array}$} \\
\hline QHU36824.1:Spike_China(1273) & & \\
\hline BCB15090.1:spike_Japan(1273) & & \\
\hline QHR63250.2:spike_China(1273) & & \\
\hline QHR84449.1:Spike:Australia(1273) & S247R & \\
\hline QHS34546.1: Spike:India(1272) & Y145- & R407I \\
\hline QHW06059.1:Spike:USA:CA(1273) & H49Y & \\
\hline QHZ00379.1:Spike:South Korea(1273) & S221W & \\
\hline QIA20044.1:Spike:China Yunnan(1273) & Y28N & \\
\hline QIA98554.1:spike_ttaly(1273) & & \\
\hline QIA98583.1:Spike: India(1273) & A929V & \\
\hline QIB84673.1:spike_Nepal(1273) & & \\
\hline QIC53204.1:Spike:Sweden(1273) & F797C & \\
\hline QIG55994.1:spike_Brazi(1273) & & \\
\hline QIK50427.1: Spike:USA:CA(1273) & D614G & \\
\hline QIK50448.1:spike_VietNam(1273) & & \\
\hline QIQ08790.1:spike_Spain(1273) & & \\
\hline QIQ50172.1:Spike:USA:WA(1273) & D614G & \\
\hline
\end{tabular}

b

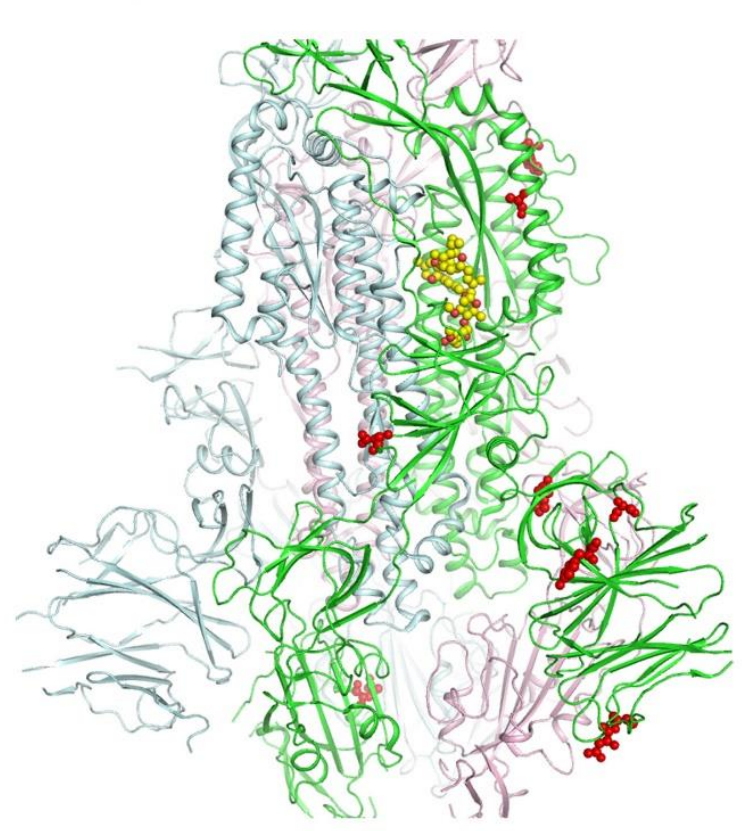

Figure 4: (a) Mutations in SARS-CoV-2 spike glycoprotein with respect to China Wuhan isolate are tabulated; (b) mutations are mapped in the structure of SARS-CoV-2 spike glycoprotein in closed state (PDB code: 6VXX) where mutated amino acids are shown in red and ivermectin is shown in ball and stick (carbon:yellow, oxygen:red). 